\title{
Group Representations and Creation Operators
}

\author{
ROBert HermanN* \\ Applied Mathematics Division, Argonne National Laboratory
}

Received November 20, 1965

\begin{abstract}
A general formalism for describing unitary representations of noncompact semisimple groups is presented. It is applied to describe some general properties of representations of $S U(6,6)$, of possible interest for the classification of elementary particles.
\end{abstract}

\section{Introduction}

Recent speculation (e.g. see [2]) has suggested that Lie group theory (particularly the theory of infinite dimensional unitary representations) will play a role in classifying elementary particles. Of course, this must follow experiment, since there is little general theory beyond the general principles of quantum mechanics. Presumably, future discoveries of particles will reveal supermultiplets of new groups and possibly infinite supermultiplets defined by representations of non-compact groups, just as experimental activity of the past 15 years has revealed the $S U(3)$ symmetry. (The experimental situation at the moment seems not sufficiently developed to proceed with confidence very far „,beyond” $S U(3)$, although of course the $S U(6)$ activity has had a few striking successes.)

At any rate, these exciting possibilities suggest development of parts of the theory of Lie groups that have been hardly touched by mathematicians. For example, one basic question can be described as follows: Given a sequence of integers (which are, say, dimensions of various multiplets of particles) can one find groups $K$ and $G$, with $K$ a subgroup of $G$, and an irreducible unitary representation of $G$ which admits subspaces invariant under $K$ of precisely this dimension? In turn, this suggests a program of classifying representations of groups by prescribing which representations of subgroups (say, the maximal compact subgroup) may occur in the representation.

In this paper (hopefully, the first in a series) we treat a number of topics that fit into this program, particularly emphasizing (following Feymmann and GeLL-ManN [2]) the need for generating representations by "creation and annihilation operators". For background on notations, etc., refer to $[4,5]$.

\footnotetext{
* This work was supported by the office of Naval Research NONR 3656/69.
} 
I would particularly like to thank J. Feldman, H. Harari and E. STEIN for help with this material.

\section{Creation and annihilation operators}

Let $H$ be a (complex) Hilbert space that is a direct sum $H^{0}+H^{1}+\cdots$ of orthogonal subspaces. Suppose that $V$ is another vector space. Suppose given a bilinear mapping $V \times H \rightarrow H$ such that:

The image of $V \times H^{r}$ lies in $H^{r+1}, r=0,1, \ldots$ Denote elements of $H$ by $\psi, \psi^{\prime}$, etc. Denote the inner product by $\left\langle\psi \mid \psi^{\prime}\right\rangle$, the norm by $\|\psi\|=\sqrt{\langle\psi \mid \psi\rangle}$.

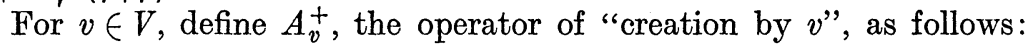

For $\psi \in H^{r}, A_{v}^{+}(\psi)$ is the image in $H^{r+1}$ or $(v, \psi)$. Thus $V_{v}^{+}$maps $H^{r}$ into $H^{r+1}$. We can now define $A_{v}^{-}$, the operator of , annihilation by $v^{\text {" }}$ as the Hermitian adjoint of $A_{v}^{+}$:

$$
\left\langle\psi \mid A_{v}^{+} \psi^{\prime}\right\rangle=\left\langle A_{v}^{-} \psi \mid \psi^{\prime}\right\rangle
$$

Note that $A_{v}^{-}$maps $H^{r}$ into $H^{r-1}$. We can now define the skew-Hermitian operator $A_{v}=A_{v}^{+}-A_{v}^{-}$. We require that for $v, v_{1} \in V, A_{v}^{+}$commutes with $A_{v_{1}}^{+}$. After taking adjoints, we see that this implies that two annihilation operators commute. Hence,

$$
\left[A_{v}, A_{v_{1}}\right]=\left[A_{v_{1}}^{-}, A_{v}^{+}\right]+\left[A_{v_{1}}^{+}, A_{v}^{-}\right] .
$$

In particular, $\left[A_{v}, A_{v_{1}}\right]$ is a skew-Hermitian operator on $H$ mapping $H^{r}$ into $H^{r}$.

This suggests that we construct the Lie algebra $\mathbf{G}$ of skew-Hermitian operators generated by the $\left\{A_{v}: v \in V\right\}$. If $G$ is a group whose Lie algebra is $\mathbf{G}$, and if the representation of $\mathbf{G}$ extends to a representation of $G$, we have obviously a unitary representation of $G$. We can now let $K$ be the subgroup of elements of $G$ that map $H^{r}$ into $H^{r}$, for $r=0,1,2, \ldots$, and $\mathbf{K}$ the corresponding Lie algebra of operators. Let $\mathbf{P}$ be the subspace of operators of $\mathbf{G}$ spanned by the operators $\left\{A_{v}: v \in V\right\}$. Thus, $[\mathbf{P}, \mathbf{P}] \subset \mathbf{K}$. We see that $K$ has a structure very much like that of a symmetric subgroup of a Lie group. In turn, we will see in the next section how symmetric subgroups generate this sort of structure.

A useful modification can be made in the definition of $A_{v}$. Suppose also that, for $v \in V, A_{v}^{0}$ is a skew-Hermitian operator on $H$ which maps $H^{r}$ into $H^{r}$. We can modify $A_{v}$ as:

$$
A_{v}=A_{v}^{+}-A_{v}^{-}+A_{v}^{0} \text {. }
$$

The Fock space construction $[1,7]$ in quantum field theory is one typical case of this construction, which suggests that the more general forms might be useful in elementary particle physics. Cook [1] has shown that the operators $i A_{v}$ are self-adjoint in the technical sense of 
Hilbert space theory, thus providing a firm analytical foundation for the theory of free fields, at least. In Section 5 we will give a new easy proof of this fact, and show that with natural assumptions it extends to more general situations.

\section{Creation operators generated by unitary representations}

Our main goal is to construct interesting unitary representations using the creation operators as a beginning. However, we can more easily show conversely that unitary representations give creation operators. For this purpose, we will sketch an approach to the problem of classifying unitary representations that has been suggested and studied by THIELEKER in his doctoral dissertation [9].

Suppose that $\mathbf{G}$ is a Lie algebra of skew-Hermitian operators on a Hilbert space $H$, and that $\mathbf{K}$ and $\mathbf{P}$ are subspaces of $G$ such that:

$$
\mathbf{G}=\mathbf{K} \oplus \mathbf{P} ;[\mathbf{K}, \mathbf{K}] \subset \mathbf{K} ;[\mathbf{K}, \mathbf{P}] \subset \mathbf{P} ;[\mathbf{P}, \mathbf{P}] \subset \mathbf{K} .
$$

( $K$ is then called a symmetric subalgebra of $\mathbf{G}$ ). Suppose $H^{0}$ is a subspace of $H$ which is invariant and irreducible undre the action of $K$; let us call this representation $\sigma$. If $G$ acts irreducibly the whole representation can be built up out of products of the form:

$$
X_{1} \ldots X_{r}(\psi), X_{1}, \ldots, X_{r} \in \mathbf{P}, \psi \in H^{0} .
$$

Further, in such products we can regard the $X$ 's as only occurring in their symmetric combinations. since $[\mathbf{P}, \mathbf{P}] \subset \mathbf{K}$, and commutators of $\mathbf{P}$ can be moved to the right and be absorbed in $H^{0}$. The action of $\mathrm{Ad} \mathbf{K}$ in $\mathbf{P}$ induces a representation that we can denote by $\operatorname{Ad}_{\mathbf{P}} \mathbf{K}$. Thus, the $K$-content of the representation is obtained by decomposing into irreducible representations the representations:

$$
\sigma, \sigma \otimes \operatorname{Ad}_{\mathbf{P}} \mathbf{K}, \operatorname{Ad}_{\mathbf{P}} \mathbf{K} \circ \operatorname{Ad}_{\mathbf{P}} \mathbf{K} \otimes \sigma, \quad \text { etc., }
$$

where $\otimes$ denotes the tensor product and $\circ$ denotes the symmetric tensor product of two identical representations. Further details of this argument will be found in Thieleker's work.

Of course, one still has to decompose the representations given in (3.3) into irreducible components. Certain representations that abstractly might occur in this decomposition in reality will not occur in the decomposition of $\mathbf{K}$ on $H$. For example, consider a Casimir operator $D$ of $\mathbf{G}$, i.e. a polynomial in the generators of $\mathbf{G}$ that commutes with the action of AdG. Since the representation of $G$ on $H$ is irreducible, $D$ will be a scalar on $H$. Using the commutation relations (3.1) $D$ can be written as the sum of products of the form $X_{1} \ldots X_{r} Y_{1} \ldots Y_{s}$, with the $X$ 's in $\mathbf{P}$, the $Y$ 's in $\mathbf{K}$. We see immediately that the fact that this is to be a scalar will give certain relations among the tensor products (3.3).

There is also a relation between this approach and the global methods of GELFAND and NatMaRK, which involves finding homogeneous spaces 
of $G$ on which $K$ also acts transitively. For example, if $G$ is semisimple and if $K$ is the maximal compact subgroup, the orbit of $K$ on an element of $\mathbf{P}$ will be such a homogeneous space. Now, talking about the,,symmetric tensor products" of $\operatorname{Ad}_{\mathbf{P}} \mathbf{K}$ is just a fancy way of talking about the action of $K$ on the polynomial functions on the vector space P. One will obtain representations of $K$ that fit together into unitary representations of $G$ by considering the action of $K$ on such polynomial functions after restricting them to an orbit of $K$. Certain of these polynomials will be constant on the orbit of $K$, which should correspond, in favorable cases, to the relations among the tensor products that we derived from the Casimir operators of $\mathbf{G}$ using Thieleker's algebraic approach.

Now we can describe the relevance of this to the creation-annihilation operator formalism. Define the representation Hilbert space $H$ as a direct sum of orthogonal subspaces $H^{0}+H^{1}+\cdots$ in the following way:

$H^{0}$ is as defined above, $H^{1}$ is the orthogonal complement of $H^{0}$ in the subspace of $H$ spanned by $H^{0}$ and the set of vectors $\{X(\psi): X \in P$, $\psi \in H\} . H^{2}$ is the orthogonal complement of $H^{0}+H^{1}$ in the subspace spanned by $H^{0}, H^{1}$ and the vectors $\left\{X_{1} X_{2}(\psi): X_{1}, X_{2} \in P, \psi \in H^{0}\right\}$. Continue by induction to define $H^{r}$.

To provide the setup described, ,axiomatically" in Section 2, take $V$ as $\mathbf{P}$, and define the bilinear map $V \times H^{r} \rightarrow H^{r+1}$ used to define creation operators in the following way:

Assign to $X \in \mathbf{P}, \psi \in H$, the projection in $H^{r+1}$ of $X(\psi)$. Having defined annihilation and creation operators in this way from the representation one might ask conversely how the operators of $\mathbf{P}$ may be expressed in terms of them.

Lemma 3.1. For $\psi \in H^{r}, A_{\bar{X}}(\psi)=-$ (the projection of $X(\psi)$ in $H^{r-1}$ ).

Proof. Since $A_{X}^{+}$maps $H^{r}$ into $H^{r-1}, A_{\bar{X}}$, its adjoint, maps $H^{r}$ into $H^{r-1}$. For $\psi \in H, \psi^{\prime} \in H^{r-1}$,

$$
\left\langle\psi^{\prime} \mid A_{\bar{X}} \psi\right\rangle=\left\langle A_{X}^{+} \psi^{\prime} \mid \psi\right\rangle=\left\langle X \psi^{\prime} \mid \psi\right\rangle=\left\langle X \psi^{\prime} \mid \psi\right\rangle=\left\langle\psi^{\prime} \mid X \psi\right\rangle
$$

which proves 3.1 .

Lemma 3.2. $\left[A_{X}^{+}, A_{Y}^{+}\right]=0=\left[A_{\bar{X}}, A_{\bar{Y}}\right]$ for $X, Y \in \mathbf{P}$.

Proof. $A_{X}^{+} A_{Y}^{+}(\psi)$, for $\psi \in H^{r}$, is obviously the projection of $X Y(\psi)$ on $H^{r+2}$, which is also the projection of $Y X(\psi)$ on $H^{r+2}$, since $[X, Y] \in K$.

Now, for $X \in P, \psi \in H^{r}$, define $A_{X}^{0}(\psi)$ as the projection of $X(\psi)$ on $H^{r}$. Then, we see that operator

$$
A_{\bar{X}}^{+}+A_{X}^{0}-A_{\bar{X}}^{-}
$$

satisfies the same commutation relations as does $X$ : Assigning this operator to $X$ then defines another skew-Hermitian representation of $G$, which is identical to the original one only if

$$
X\left(H^{r}\right) \subset H^{r+1}+H^{r}+H^{r-1} .
$$




\section{Another general method for constructing creation operators}

Let $\mathbf{K}$ be a Lie algebra that is either semisimple or the direct sum of a semisimple and an abelian Lie algebra. Let $\mathbf{C}$ be a Cartan subalgebra of $\mathbf{K}$, i.e. a maximal abelian subalgebra such that for $X \in \mathbf{C}, \operatorname{Ad} X$ is diagonalizable as a transformation on $\mathbf{G}$.

Consider an irreducible linear representation of $\mathbf{K}$ on a finite-dimensional complex vector space $V$. The transformations of $\mathbf{C}$ can be simultaneously diagonalized; the eigenvalues as linear forms on $\mathbf{C}$, are the weights of the representation. A partial ordering can be introduced into the vector space over the real numbers generated by all possible weights of all representations. There is just one weight of the given representation that is maximal relative to this ordering, and this maximal weight serves to characterize the representation. Further, the maximal weights form a lattice generated by $c$ (= dimension $\mathbf{C}$ ) "fundamental" maximal weights. (All these facts are well-known in Lie algebra theory: see JACOBSON's book [6] or the summary given in [4].)

Suppose given representations of $\mathbf{K}$ on vector spaces $V_{1}$ and $V_{2}$, with maximal weights $w_{1}$ and $w_{2}$. One can form the tensor product representation of $\mathbf{K}$ on $V_{1} \otimes V_{2}$. It will be reducible. However, another general theorem of representation theory asserts that there will be exactly one representation of $\mathbf{K}$ in this tensor product with maximal weight $w_{1}+w_{2}$. Let us denote this subspace of the tensor product by $V_{3} . V_{3}$ then results from combining the representations of $K$ on $V_{1}$ and $V_{2}$ in a definite way; we may call this the Cartan product of the two representations. This method of construction provides a bilinear map $V_{1} \times V_{2} \rightarrow V_{3}$ that commutes with the action of $\mathbf{K}$ on each space.

Let us apply this construction to build a Hilbert space $H=H^{0}+H^{1}+\cdots$ with creation operators in the following way: choose $H^{0}$ as $V_{1}, V$ as $V_{2}, H^{1}$ as $V_{3}$, the Cartan product of $V_{1}$ and $V_{2}$. Similiarly, let $H^{2}$ be the Cartan product of $H^{1}$ and $V_{2}$, whose maximal weight is then $w_{1}+2 w_{2}$, etc. The bilinear map $H^{r} \times V_{2} \rightarrow H^{r+1}$ thus defines the creation operators.

This construction is a generalization of the Fock space construction for bosons. (In fact, we obtain precisely this if we take $K$ as $U(n), V_{1}$ and $V_{2}$ as the one and $n$-dimensional representations of $K$. One obtains the Fock space for fermions by choosing $K, V_{1}$ and $V_{2}$ as the same, but modifying the construction by requiring that the bilinear map $H^{r} \times V_{2} \rightarrow H^{r+1}$ be that which picks out the representation in the tensor product $H^{r} \oplus V_{2}$ that has minimal maximal weight. Again, there is just one such irreducible representation in the tensor product.)

An interesting point about this construction and its possible usefulness in physics is that it bears some resemblance to the procedure physicists use heuristically to build up particles from basic ones, such as "quarks". 
A short glance at the examples should convince the reader that the "degenerate series" of unitary representations of such groups as $S L(n, C)$, $S O(n, 1)$ come within this construction.

\section{Analytic vectors}

We have given methods for constructing representations of Lie algebras by means of annihilation and creation operators. It is wellknown that this "infinitesimal" approach does not always lead to global representation of the corresponding simply connected Lie groups. For example, in the pioneering work of WIGNER and BARGMANN one notices a certain amount of healthy caution as to this sort of pathology. However, a strong technique for overcoming these difficulties in most of the situations of practical interest has been introduced by HARISH-CHANDRA with the notion of "analytic vector". NELSON took up this idea, and in a definitive and powerful work [8] constructed very firm foundations. In this section we will sketch how these ideas apply in a very natural way to the situations of interest in this paper.

Let $H$ be a Hilbert space. Denote the inner product in $H$ by $\left\langle\psi \mid \psi^{\prime}\right\rangle$, and the norm by $\|\psi\|$. Let $A_{1}, \ldots, A_{n}$ be a collection of operators defined on domains in $H$. A vector $\psi$ in $H$ is an analytic vector for these operators if:

a) $\psi$ lies in the domain of the algebra of operators generated by $A_{1}, \ldots, A_{n}$.

b) $\sum_{j=0}^{\infty} \frac{t^{2}}{j}\left\|\left(A_{1}+\cdots+A_{n}\right)^{j}(\psi)\right\|<\infty$ for some $t>0$.

We will not attempt to summarize Nelson's results here, beyond saying that if a collection of operators has a dense set of analytic vectors most of the possible pathology is ruled out and much the same results hold as for operators in finite dimensional spaces, particulary concerning the question whether a Lie algebra of operators generates a global group of transformations. As an example, we will describe one of the simplest theorems:

Consider an Hermitian operator $A$ defined on a domain $D(A)$ in $H$. Suppose that $A$ is a closed operator. Then, $A$ is self-adjoint if and only if $D(A)$ contains a dense set of analytic vectors for $A$.

( $A$ is closed if it has the following property: Suppose $\psi_{1}, \psi_{2}, \ldots$ is a sequence of elements of $D(A)$ such that $\lim _{j \rightarrow \infty} \psi_{j}=\psi$ and $\lim _{j \rightarrow \infty} A \psi_{2}=\psi_{i}$. Then, we require that $\psi \in D(A)$ and $\psi^{\prime}=A \psi$. Any Hermitian operator can be extended in a unique way to give a minimal closed one. ,Selfadjoint" is taken in the technical sense of Hilbert space theory. For the purposes of both representation theory and physics the following property of self-adjoint operators is the important one: 
An Hermitian operator on a domain $D(A)$ is self-adjoint if and only if there is a one-parameter group $t \rightarrow U(t)$ of unitary transformations on $H$ of which $A$ is the infinitesimal generator, i.e. $\psi \in D(A)$ if and only if $\lim _{t \rightarrow 0}(U(t) \psi-\psi) / t$ exists and the limit is precisely $i A(\psi)$.)

Now, let us consider the Hermitian operators constructed from annihilation and creation operators according to the scheme described in Section 2.

Let $H$ be a Hilbert space and let $H^{0}, H^{1}, \ldots$ be a sequence of mutually orthogonal subspaces of $H$ whose direct sum is dense in $H$. Let $A^{+}$ be an operator defined at least on each of these subspaces which maps $H^{r}$ into $H^{r+1}, r=0,1, \ldots$ Let $A^{-}$be its adjoint, and let $A=\frac{1}{2}\left(A^{+}+A^{-}\right)$. Our goal is to find conditions that guarantee that each of the vectors of $H^{r}, r=0,1, \ldots$, is an analytic vector for $A$, hence that the minimal closed operator defined by $A$ be self-adjoint. For simplicity, we are just considering the case of one such operator; a collection of such operators can be treated with the same methods.

For $r=0,1, \ldots$, let $a_{r}$ be the norm of $A^{+}$restricted to $H^{r}$. Thus, $\left\|A^{+} \psi\right\| \leqq a_{r}\|\psi\|$ for $\psi \in H^{r}$, and $a_{r}$ is the smallest such number.

Lemma 5.1. The norm of $A^{-}$on the subspace $H^{r}$ is no greater than $a_{r-1}$.

Proof. Let $\psi \in H^{r}$ have norm 1. Using the Schwarz inequality,

$$
\left\|A^{-} \psi\right\|^{2}=\left\langle\psi \mid A^{+} A^{-} \psi\right\rangle \leqq\left\|A^{+} A^{-} \psi\right\| \leqq a_{r-1}\left\|A^{-} \psi\right\| .
$$

Lemma 5.1 follows after dividing by $\left\|A^{-} \psi\right\|$.

$$
\begin{gathered}
\text { Now, }\|A \psi\|^{2}=\frac{1}{4}\left\|A^{+} \psi+A^{-} \psi\right\|^{2}=\frac{1}{4}\left(\left\|A^{+} \psi\right\|^{2}+\left\|A^{-} \psi\right\|^{2}\right) \leqq \\
\leqq \frac{1}{4}\left(a_{r}^{2}+a_{r-1}^{2}\right), \text { whence } \\
\|A \psi\| \leqq \max \left(a_{r}, a_{r-1}\right) .
\end{gathered}
$$

Now let us turn to finding conditions that the elements of the $H^{r}$ be analytic vectors for $A$. Suppose that $a_{n},{ }_{r}$ is the norm of $A^{n}$ in the subspace $H^{r}$. We must estimate its value. Let $b_{r}$ be the maximum among the numbers $a_{0}, a_{1}, \ldots, a_{n}$.

$$
\begin{aligned}
& \left\|A^{n} \psi\right\|=\left\|A^{n-1}\left(\frac{1}{2}\left(A^{+} \psi+A^{-} \psi\right)\right)\right\| \leqq \\
& \leqq \frac{1}{2}\left(a_{n-1}, r+1 a_{r}+a_{n-1}, r_{r-r 1} a_{-1}\right) \leqq \\
& \leqq \frac{1}{2}\left(b_{r}\left(a_{n-1}, r+1+a_{n-1}, r-1\right)\right. \text {, hence: } \\
& a_{n}, r \leqq \frac{1}{2} b_{r}\left(a_{n-1}, r+1+a_{n-1, r-1}\right) \text {. }
\end{aligned}
$$


From (5.1), we have: $a_{1, r} \leqq b_{r}$. Thus,

$$
\begin{aligned}
a_{2}, r & \leqq \frac{1}{2} b_{r}\left(b_{r+1}+b_{r-1}\right) \leqq b_{r} b_{r+1} . \\
a_{3, r} & \leqq \frac{1}{2} b_{r}\left(a_{2}, n+1\right. \\
& \left.\leqq \frac{1}{2} b_{2} b_{r-1}\right) \leqq \frac{1}{2} b_{r+1} b_{r+2} .
\end{aligned}
$$

Continuing by induction, we see that:

and

$$
a_{n, r} \leqq b_{r+n-1} b_{r+n-2} \ldots b_{r}
$$

$$
\sum_{n} \frac{\left\|A^{n} \psi\right\| t^{n}}{n !} \leqq \sum_{n} \frac{a_{n, r}\|\psi\| t^{n}}{n !} \leqq \sum_{n} \frac{\left(b_{r+n-1} b_{r+n-2} \ldots b_{r}\right) t^{n}}{n(n-1) \cdots} .
$$

Applying the Ratio Test, this will converge if:

$$
\lim _{n \rightarrow \infty} \frac{b_{r+n-1}}{n}<\frac{1}{t},
$$

or

Thus, we have proved:

$$
\lim _{r \rightarrow \infty} \frac{b_{r}}{r}<\frac{1}{t} .
$$

Theorem. Let $a_{r}$ be the norm of $A^{+}$on the subspace $H^{r}$ of $H$. Let $b_{r}$ be the maximum among the numbers $a_{0}, \ldots, a_{n}$. If $b_{r} / r$ is bounded as $r \rightarrow \infty$, then each vector of the subspace of $H$ which is the union of $H^{0}$, $H^{0}+H^{1}, \ldots$, is an analytic vector for $A$. If further $b_{r} / r \rightarrow 0$ as $r \rightarrow \infty$ then the series $\Sigma \frac{t^{n} A^{n} \psi}{n}$ has an infinite radius of convergence for $\psi \in H^{r}$.

We now show how this applies to the annihilation and creation operators in the Fock space construction. For simplicity, let us consider the boson case. (In fact, these operators are bounded in the fermion case anyway; hence the exponential series always converges for any vector in $H$.) $H^{1}$ is then an arbitrary Hilbert space, $H^{0}$ is one dimensional (the "vacuum state"), and $H^{r}$ (the "many particle states") is the $r$-fold symmetric tensor product of $H^{1}$, i.e. is spanned by elements of the form $\psi_{1} \circ \cdots \circ \psi_{r}$, with $\psi_{1}, \ldots, \psi_{r} \in H^{1}$. ( $\circ$ denotes the symmetric tensor product.) The inner product in $H^{r}$ is determined by the following formula:

$$
\left\langle\psi_{1} \circ \cdots \circ \psi_{r} \mid \psi_{1}^{\prime} \circ \cdots \circ \psi_{r}^{\prime}\right\rangle=\frac{1}{r !} \sum\left\langle\left.\psi\right|_{1} \psi_{r}^{\prime}\right\rangle \cdots\left\langle\psi_{r} \mid \psi_{j r}\right\rangle ;
$$

where the sum is over all permutations $(1, \ldots, r) \rightarrow\left(i_{1}, \ldots, i_{r}\right)$ of the set of $r$ integers. In particular, if $\psi_{1}, \ldots, \psi_{r}$ are vectors from an orthonormal basis of $H^{1}$, then $\left\|\psi_{1} \circ \cdots \circ \psi_{r}\right\| \leqq 1$ and is equal to 1 if $\psi_{1}=\psi_{2}=\cdots=\psi_{r}$. It is readily seen $[1,7]$ that, for $\psi \in H^{1}, A_{\psi}^{+}$and $A_{\psi}^{-}$ 
are defined as follows:

$$
\begin{aligned}
A^{+}\left(\psi_{1} \circ \cdots \circ \psi_{r}\right) & =\sqrt{r+1} \psi \circ \psi_{1} \circ \cdots \circ \psi_{r} \cdots \\
A^{-}\left(\psi_{1} \circ \cdots \circ \psi_{r}\right) & =\frac{1}{\sqrt{r}}\left(\left\langle\psi \mid \psi_{1}\right\rangle \psi_{2} \circ \cdots \circ \psi_{r}+\cdots+\left\langle\psi \mid \psi_{r}\right\rangle\right. \\
& \left.\psi_{1} \circ \cdots \circ \psi_{r-1}\right) .
\end{aligned}
$$

We see then that the norm of $A^{+}$restricted to $H^{r}$ is $(r+1)^{\frac{1}{2}}$, hence $b_{r}$ is also $(r+1)^{\frac{1}{2}}$. The theorem applies; in fact, since $b_{r} / r \rightarrow 0$, the radius of convergence of the exponential series is infinite. In particular, the operators $A_{\psi}=\frac{1}{2}\left(A_{\psi}^{+}+A_{\psi}^{-}\right)$are self-adjoint after closure, which is Cook's theorem [1]. Cook also deals with the case of "mixed statistics". In fact, it is readily seen that the norm of $A^{+}$is greatest in the boson case, so that the analytic vector argument applies in the "mixed statistics" case also. In the fermion case, $b_{r}=1$, so that $A_{\psi}$ is a bounded operator and the exponential series converges for any $\psi$ in $H$.

\section{Discrete series and symmetric bounded domains}

Let $G$ be a connected semisimple non-compact Lie group with finite center and let $K$ be a maximal compact subgroup of $G$. Then $\mathbf{G}$, the Lie algebra of $G$, can be split up by the Cartan decomposition:

$$
\mathbf{G}=\mathbf{K} \oplus \mathbf{P},[\mathbf{K}, \mathbf{P}] \subset \mathbf{P},[\mathbf{P}, \mathbf{P}] \subset \mathbf{K} .
$$

We will suppose that the situation is "irreducible" in the sense that the adjoint representation of $K$ in $\mathbf{P}$, that we denoted in Section 3 by $\operatorname{Ad}_{\mathbf{p}} K$, is irreducible over the real numbers. (It may be reducible over the complex numbers; in fact, this is precisely the situation of interest in this section.) It is then known [3] that either:

a) $\mathbf{G}$ and $\mathbf{G}_{c}$ are simple Lie algebras or

b) $\mathbf{G}$ is the complex Lie algebra $\mathbf{K}_{c}$, and $\mathbf{G}_{c}$ is isomorphic to the direct sum of two copies of $\mathbf{K}_{\mathbf{c}}$.

$\left(\mathbf{G}_{\mathbf{c}}\right.$ denotes the "complexification" of $\mathbf{G}$, i.e. $\mathbf{G}=\mathbf{G}+\mathbf{i} \mathbf{G}$, which may be viewed either as a Lie algebra over the real or complex numbers.)

Examples of a) are such groups as $S U(m, n), S O(m, n)$, while examples of b) are $G=S O(n, C), S L(n, C)$. In this section, we will only be concerned with situation a). Here there are again two main classes:

A. $\mathbf{K}$ is semisimple.

B. $\mathbf{K}$ is not semisimple, i.e $\mathbf{K}$ is the direct sum of a semisimple ideal $\mathbf{K}_{S}$ and a center, which can be proved to be one-dimensional, generated, say, by an element $Z$.

Examples of type A are: $G=S O(n, 1), K=S O(n) ; G=S U(n)$, $K=S O(n)$. Type B: $G=S L(2, R), K=S O(2, R) ; G=S U(m, n)$,

$$
\begin{aligned}
K & =S U(m) \times S U(n) \times U(1) ; \\
G & =S O(m, 2), K=S O(m) \times S O(2) .
\end{aligned}
$$


The groups of type B have simply connected covering groups with infinite center. (Such groups have no faithful finite dimensional representations, however.)

This distinction between types A and B becomes important when one tries to classify particles by choosing infinite dimensional unitary representations of $G$. Assuming that $K$ is the exact or approximate symmetry (in the sense, say, that it commutes or nearly commutes with the strongest part of the Hamiltonian, whereas the operators of $\mathbf{P}$ have something to do with the interactions between multiplets) we see that in the type B case the center of $K$ provides an additional additive quantum number that commutes with all the other operators of $K$. For example, one might try to use $K=S U(3) \times U(1)$, with the $S U(3)$ part of $K$ giving the Eightfold Way classification, while the $U(1)$-part gives something like the baryon number.

On the mathematical side, the representations of groups of type B have a special feature, namely that they have certain "isolated" representations which are called the "discrete series". (The name arises from the role they play in the Plancherel formula, which will not concern us here.) We will now describe the relevance of these discrete series to the physical questions, relying on the ideas described in Section 3.

Suppose from now on that $G$ is of type $B$, with $Z$ the generator of the center of $K$. Now, $\operatorname{Ad} Z$ acting on $\mathbf{P}$ commutes with the action of $\operatorname{Ad} K$. Let us denote this transformation of $P$ by $J$, i.e.

$$
J X=[Z, X] \text { for } X \in \mathbf{P} .
$$

Since $\exp (t Z)$ lies in a compact group, $J$ has pure imaginary eigenvalues, which must be non-zero. Since $J$ is a real transformation the negative of an eigenvalue is also an eigenvalue. Thus, $\mathbf{P}_{c}=\mathbf{P}+i \mathbf{P}$ splits up into the direct sum of two complex subspaces $\mathbf{P}^{+} \oplus \mathbf{P}^{-}$defined by the eigenvalues $i a$, with $a>0$ and $a<0$ respectively. Each of these subspaces is isomorphic to $\mathbf{P}$. If there were two eigenvalues with positive imaginary part, Ad $K$ acting in $\mathbf{P}$ would not be irreducible. Then by normalizing $Z$ we can arrange that the eigenvalues of $J$ are $\pm i$. In particular,

$$
J^{2}=-I \text {. }
$$

We can use (6.1) directly to make $\mathbf{P}$ into a complex vector space. (This is also implicit in the isomorphism between $\mathbf{P}$ and $\mathbf{P}^{+}$.) Namely, for a complex scalar $a+b i$, with $a$ and $b$ real, define, for $X \in P$ :

$$
(a+b i) X=a X+b J X .
$$

This way of considering $\mathbf{P}$ as a complex vector space assures us that $\operatorname{Ad} K$ acts in $\mathbf{P}$ as a group of complex transformations; notice that $J$ acts by multiplication by $i$. 
Lemma 6.1. Let $\mathbf{P}^{+}=\{X-i J X: X \in \mathbf{P}\}$ and $\mathbf{P}^{-}=\{X+i J X: X \in \mathbf{P}\}$. Then, $\mathbf{P}^{+}$and $\mathbf{P}^{-}$are, respectively, the $i$ and $-i$ eigenspaces of $J$. Further, $\left[\mathbf{P}^{+}, \mathbf{P}^{+}\right]=0=\left[\mathbf{P}^{-}, \mathbf{P}^{-}\right]$.

Proof. That $\mathbf{P}^{+}$is the $i$-eigenspace follows from:

Let $X, Y \in \mathbf{P}$.

$$
J(X-i J X)=J X+i X=i(X-J X) .
$$

$$
\begin{aligned}
{[X-i J X, Y-i J Y] } & =[X, Y]-[J X, J Y]-i([J X, Y]+[X, J Y]) \\
{[J X, J Y]=[[Z, X],} & {[Z, Y]]=[Z,[X,[Z, Y]]]-[X,[Z,[Z, Y]]] } \\
& =-\left[X, J^{2} Y\right]=[X, Y] . \\
{[J X, Y] } & =[Z, X], Y]=[Z,[X, Y]-[X,[Z, Y]] \\
& =-[X, J Y] .
\end{aligned}
$$

These three identities show that $\left[\mathbf{P}^{+}, \mathbf{P}^{+}\right]=0$. Similiarly, $\left[\mathbf{P}^{-}, \mathbf{P}^{-}\right]=0$.

Now let us suppose that $\mathrm{G}$ is a Lie algebra of skew-Hermitian operators on a Hilbert space $H$. Let us build up $H$ as a direct sum $H^{0}+H^{1}+\cdots$ of subspaces invariant under $K$, as explained in Section 3. Further, suppose that

$$
\text { dimension } H^{0}=1 \text {. }
$$

(This type of representation seems of most immediate interest for the classification of particles.) Then, we have

$\mathbf{K}_{S} H^{0}=0$, since a semisimple Lie algebra admits no nontrivial one dimensional representations. Let us look at $H^{1}$, which is spanned by vectors of the form $\left\{X\left(\psi_{0}\right): X \in \mathbf{P}\right\}$, where $\psi_{0}$ is a fixed generator of $H^{0}$. Let $\sigma$ denote the representation of $\operatorname{Ad} K$ in $\mathbf{P}$, considering $\mathbf{P}$ as a complex vector space. Let $\bar{\sigma}$ denote the complex conjugate representation. Then, there are the following alternatives for the representation of $K$ in $H^{1}$ :

\section{Either}

(A) $H^{1}$ splits into parts invariant under $K$, in one of which $K$ acts by $\sigma$, in the other by $\bar{\sigma}$, or

(B) $H^{1}$ is irreducible undre the action of $K$, and either $\mathbf{P}^{+}$or $\mathbf{P}^{-}$ (but not both) annihilate $\psi_{0}$. $K$ acts via $\sigma$ or $\bar{\sigma}$.

Proof, Consider the mapping $\mathbf{P}_{c} \rightarrow H^{1}$ which sends $X+i Y$ into $(X+i Y)\left(\psi_{0}\right)$. It is clearly onto $H^{1}$, and commutes with the action of $K$ on both spaces. Hence, the kernel is invariant under $\operatorname{Ad} K$. But, we have seen that the only such spaces of $\mathbf{P}_{c}$ are $\mathbf{P}^{+}$and $\mathbf{P}^{-}$, and one goes into the other under complex conjugation. (A) applies if the kernel is zero, (B) if it is non-zero.

For the physical applications one is interested in representations in which the representations of $K$ appear the fewest number of times. Thus, the representations satisfying (B) seem to be the most interesting 
candidates to investigate further. We will suppose from now on that

$$
\mathbf{P}^{+} \psi_{0}=0 \text {. }
$$

Consider that action of $Z$ on $\psi_{0}$. We must have

$$
Z \psi_{0}=i \alpha_{\psi_{0}}, \text { for some real } \alpha \text {. }
$$

If this is to be a global representation of $G, \alpha$ can have only a discrete set of values. For, suppose that $t_{0}$ is the first positive value of $t$ for which $\exp \left(t_{0} Z\right)=$ identity. Then,

$$
\alpha t_{0} \text { is a multiple of } 2 \pi \text {. }
$$

There is another relation provided by the second order Casimir operator of $\boldsymbol{G}$. Let $B(X, Y)$ be the Killing form of $\mathrm{G}$.

Lemma 6.2. For $X, Y \in \mathbf{P}$,

$$
\begin{aligned}
B(J X, J Y) & =B(X, Y) \\
B(J X, Y) & =-B(X, J Y) .
\end{aligned}
$$

Proof. As we have seen, $X-i J X$ is an eigenvactor of $\operatorname{Ad} Z$ with eigenvalue $i$. Hence,

$$
\operatorname{Ad} \exp \left(\frac{1}{2} \pi Z\right)(X-i J X)=i(X-i J X) .
$$

Taking the real and imaginary part of this relation gives:

$$
\operatorname{Ad} \exp \left(\frac{1}{2} \pi Z\right)(X)=J X .
$$

That $B(J X, J Y)=B(X, Y)$ now follows from the general fact that the Killing form is invariant under automorphisms of $G$. Now,

$$
B(X, J Y)=-B(J J X, J Y)=-B(J X, Y) .
$$

For $X \in \mathbf{P},[X+i J X, X-i J X]=2 i[J X, X] \in i K$.

For $X \in \mathbf{P}$,

$$
B([X, J X], Z)=-B(J X,[X, Z])=B(J X, J X)=B(X, X) .
$$

Since $\mathbf{P}^{+}\left(\psi_{0}\right)=0$, we have

$$
X\left(\psi_{0}\right)=i J X\left(\psi_{0}\right) \text { for } X \in P .
$$

Now, $[X-i J X, X+i J X]\left(\psi_{0}\right)=(X-i J X)(X+i J X)\left(\psi_{0}\right)$

$$
\begin{aligned}
& =X^{2} \psi_{0}+(J X)^{2} \psi_{0}-i J X X\left(\psi_{0}\right)+i X J X\left(\psi_{0}\right) \\
& =\text {, using }(6.4), 2 X^{2} \psi_{0}+(J X)^{2} \psi_{0}-i J X X \psi_{0} .
\end{aligned}
$$

But also, using (6.2), this is equal to

$i[X, J X] \psi_{0}=i\left(X J X \psi_{0}-J X X \psi_{0}\right)=$, using (6.4), $X^{2} \psi_{0}-i J X X \psi_{0}$. 
Putting these two calculations together gives:

$$
\begin{aligned}
& -2 i J X X \psi_{0}=(J X)^{2} \psi_{0}-i J X X \psi_{0}, \text { or } \\
& -i J X X \psi_{0}=(J X)^{2} \psi_{0}, \\
& i[X, J X] \psi_{0}=X^{2} \psi_{0}+(J X)^{2} \psi_{0} .
\end{aligned}
$$

Now, suppose that dimension $\mathbf{P}=2 n$. Suppose that $\left(X_{j}\right), j=1, \ldots, n$, is a set of elements of $\mathbf{P}$ such that $\left(X_{j}, J X_{j}\right)$ forms a basis for $\mathbf{P}$ that is orthonormal with respect to the Killing form, i.e.

$$
B\left(X_{j}, J X_{k}\right)=0 ; \quad B\left(X_{j}, X_{k}\right)=\delta_{j k}=B\left(J X_{j}, J X_{k}\right)
$$

(Such a basis exists because of Lemma 6.2. Recall also that $B$ is positive definite on $\mathbf{P}$, negative definite on $\mathbf{K}$.)

Suppose that $\left(Y_{u}\right), u=1, \ldots, m$, is a basis of $\mathbf{K}_{S}$ that satisfies:

$$
B\left(Y_{u}, Y_{v}\right)=-\delta_{u v} \text {. }
$$

Put $Z^{\prime}=Z-\sum_{u} B\left(Z, Y_{u}\right) Y_{u}$. Then, $\left(Y_{1}, \ldots, Y_{m}, Z^{\prime} /\left(-B\left(Z^{\prime}, Z^{\prime}\right)\right)^{\frac{1}{2}}\right.$, $\left.X_{j}, J X_{j}\right)$ is a basis of $\mathrm{G}$ that is orthonormal with respect to the Killing form. The Casimir operator $\Delta$ is given by:

$$
\Delta=\sum_{u}-Y_{u} Y_{u}+Z^{\prime 2} / B\left(Z^{\prime}, Z^{\prime}\right)+\sum_{j} X^{2}+\left(J X_{j}\right)^{2} .
$$

Suppose that $\Delta\left(\psi_{0}\right)=\lambda \psi$ for all $\psi \in H$. Let us apply this relation to $\psi_{0}$. Now, $Y_{u}\left(\psi_{0}\right)=0$, hence

Using (6.5),

$$
\left(Z^{\prime}\right)^{2} \psi_{0}=-\alpha^{2} \psi_{0}
$$

Suppose that

$$
\sum_{j} X_{i}^{2}+\left(J X_{j}\right)^{2} \psi_{0}=i \sum_{j}\left[X_{j}, J X_{j}\right]\left(\psi_{0}\right)
$$

Then,

$$
\left[X_{j}, J X_{j}\right]=\beta_{j} Z^{\prime}+\sum_{u} \gamma_{j u} Y_{u}
$$

$$
i\left[X_{j}, J X_{j}\right] \psi_{0}=-\beta_{j} .
$$

Put $\beta=-\sum_{j} \beta_{j}$. Gathering all these relations together gives:

$$
\lambda=-\alpha^{2} / B(Z, Z)+\beta_{\alpha}
$$

(This shows that the Casimir operator can have at most a discrete set of values, which is one explanation for the term "discrete series".)

We can use (6.5) to derive inequalities. Consider $X \in P$. It is a skewHermitian operator, hence $X^{2}$ is a Hermitian operator, and

Hence,

$$
\left\langle X^{2} \psi_{0} / \psi_{0}\right\rangle<0 \text {. }
$$

This gives

$$
\left\langle i\left[X_{j}, J X_{j}\right] \psi_{0} \mid \psi_{0}\right\rangle<0
$$

$$
\beta_{\alpha}<0
$$


In particular, $\alpha$ cannot be zero, i.e. $\psi_{0}$ cannot be invariant under $Z$, and $\alpha$ must have a fixed sign, independent of the representation, since $\beta$ is defined from $G$ alone. We can also regard (6.6) as a quadratic equation for $\alpha$. Since $\alpha$ is a real root, its discriminant must be positive, i.e.

$$
\beta^{2}+\frac{4 \lambda}{\beta\left(Z^{\prime}, Z^{\prime}\right)}>0 \text {. }
$$

Let us now turn from the algebraic study of these discrete series representations to a method for actually constructing them. Ultimately, one would want to perfect the algebraic method of construction using creation operators; at the moment, the geometric method which we shall use seems more efficient.

Let $\mathbf{L}$ be the subalgebra $\mathbf{K}+i \mathbf{K}+\mathbf{P}^{-}$of $\mathbf{G}_{\mathbf{c}}$. Let $G_{c}$ be the connected Lie group corresponding to $\mathbf{G}_{c}$ : It is the "complexification" of $G$. (For example, if $\left.G=S U(m, n), G_{c}=S L(m+n, C)\right)$. Let $L$ be the connected subgroup of $G_{c}$ corresponding to the subalgebra $\mathbf{L}$. It is a "complex" subgroup of $G_{c}$, i.e. is determined by complex-analytic relations, and the coset space $G_{c} / L$ is a "complex homogeneous space", i.e. local complex analytic coordinates can be introduced in $G_{c} / L$ so that the group transformations are given in these coordinates by complex-analytic functions. (See Helgason's book [3] for details about the facts described here.)

Let $P^{+}$and $P^{-}$be the connected subgroups of $G_{c}$ generated by the subalgebras $\mathbf{P}^{+}, \mathbf{P}^{-}$of $G_{c}$. They are also complex subgroups of $G_{c}$.

Let $p_{0}$ be the point of $G_{c} / L$ representing the identity coset of $L$. One can prove that:

$G \cap L=K$, i.e. the orbit of $G$ on $p_{0}$ is the coset space $G / K$, which is in fact a symmetric space. (It is called a "symmetric bounded domain". We will explain where the bounded domain comes in shortly.)

Dimensions are easily calculated:

$$
\text { real } \begin{aligned}
\operatorname{dim} G_{c} \mid L & =\text { real } \operatorname{dim} G_{c}-\text { real } \operatorname{dim} L \\
& =2 \operatorname{dim} G-2 \operatorname{dim} K-\text { real } \operatorname{dim} \mathbf{P} \\
& =\text { real } \operatorname{dim} \mathbf{P}=\operatorname{dim} G / K .
\end{aligned}
$$

This proves that the symmetric coset space, realized as the orbit $G p_{0}$, is an open subset of $G_{c} / L$.

One can also show that $P^{+} \cap L=$ the identity. Hence, real $\operatorname{dim} P^{+}$ $=$ real $\operatorname{dim} G_{c} / L$.

Which shows that the orbit $P^{+} p_{0}$ is also an open subset of $G_{c} / L$. Now, we will use two facts proved by HARISH-CHANDRA. (See [3] for proofs.)

a) $D=G p_{0}$ is contained in the orbit $P^{+} p_{0}$.

b) Complex analytic coordinates $\left(z_{1}, \ldots, z_{n}\right)$ can be introduced into the orbit $P^{+} p_{0}$, so that the operations of $P^{+}$are just translations in these 
coordinates. With respect to these coordinates $D=G p_{0}$ is realized as a bounded open subset of the space of $n$ complex variables. These complex coordinates are determined linearly by choosing a complex basis for $\mathbf{P}^{+}$, hence the action of $K$ in these coordinates is just that linear action given by $\operatorname{Ad} K$ acting in $\mathbf{P}^{+}$.

We can now construct our Hilbert space $H$ as the space of complex analytic functions $\psi(z)$ of the variable $z=\left(z_{1}, \ldots, z_{n}\right)$. The inner product is given by integration over $D$ :

$$
\left\langle\psi \mid \psi^{\prime}\right\rangle=\int_{D} \psi(z) \overline{\psi(z)} d z \overline{d z} .
$$

The action of $G$ on $H$ is now determined as follows: Let $X \in G$, and consider the one-parameter group $t \rightarrow \operatorname{Exp}(t X)$ of transformations of $D$ it generates. Represent these transformations in terms of the $z$-coordinates by:

$$
z_{j}^{\prime}=z_{j}^{\prime}(z, t)=z_{j}+t A_{j}(z)+\cdots .
$$

The vector field $\left(A_{j}^{X}\right)$ is then the infinitesimal generator of the oneparameter transformation group. In these terms, the action of $X$ on an element of our Hilbert space is:

$$
X(\psi)=\sum_{j} A_{j}^{X} \frac{\partial \psi}{\partial z_{j}}+\psi \sum_{j} \frac{\partial A_{j}^{X}}{\partial z_{j}} .
$$

For $X \in \mathbf{K}_{S}$ or $P^{+}$the term $\sum_{j} \frac{\partial A_{j}^{X}}{\partial z_{j}}$ is zero. For $X=Z$, the element of the center of $K$.

Hence,

$$
A_{j}^{Z}=i z_{j}
$$

$$
Z(1)=i n
$$

We can now let $\psi_{0}$ be the constant function $1 . \alpha$ is now $\frac{1}{2} n$. The rest of the representation can now be built up by applying the operators of $\mathbf{P}^{-}$to the "ground state" $\psi_{0}$, as explained above. The "excited state" representations with higher values of $\alpha$ are constructed along similiar lines, using homogeneous "line bundles" on $G / K$. Of course, they can also be obtained by taking tensor products of this representation with itself a sufficient number of times.

We can readily see how this applies to the case $G=S U(m, n)$, $K_{S}=S U(m) \times S U(n)$. The representation of $K_{S}$ in $\mathbf{P}^{-}$can be computed as the $(m, \bar{n})$ representation of $S U(m) \times S U(n)$, which is then also the representation of $K_{S}$ in $H^{1}$. The higher representations of $K_{S}$ in $H^{r}$ are just the symmetric tensor products of this basic one. The case $G=S U(6,6)$ is of possible great interest in physics. As mentioned in [5] (using a slightly different method) this representation is the one constructed by Feynmann and Gell-Mann [2]. 
I would like to thank N. Burgoyne and S. GLashow for their hospitality at the University of California, Berkeley.

\section{References}

[1] Cook, J. M.: Trans. Am. Math. Soc. 74, 223 (1953).

[2] Dothan, Y., M. Gell-Mann, and Y. Ne'eman: Physics Letters 17, 148 (1965).

[3] Helgason, S.: Differential geometry and symmetric spaces. New York: Academic Press 1963.

[4] Hermann, R.: Lie groups for physicists. New York: W. A. Benjamin Inc. 1965.

[5] - On representations of non-compact groups. To appear, Proceedings of Conference on Elementary Particles, Trieste (1965).

[6] Jacobson, N.: Lie algebras. New York: Wiley 1962.

[7] Kastler, D.: Introduction à l'électrodynamique quantique. Paris: Dunod 1961.

[8] Nelson, E.: Ann. Math. 70, 572-615 (1959).

[9] ThielekeR, E.: Representations of semisimple Lie groups, to appear. 